Pacific Journal of Mathematics

ON THE NUMBER OF NONPIERCING POINTS IN CERTAIN 


\section{ON THE NUMBER OF NONPIERCING POINTS IN CERTAIN CRUMPLED CUBES}

\section{ROBERT J. DAVERMAN}

Let $K$ denote the closure of the interior of a 2-sphere $S$ topologically embedded in Euclidean 3-space $E^{3}$. If $K-S$ is an open 3-cell, McMillan has proved that $K$ has at most one nonpiercing point. In this paper we use a more general condition restricting the complications of $K-S$ to describe the number of nonpiercing points. The condition is this: for some fixed integer $n K-S$ is the monotone union of cubes with $n$ holes. Under this hypothesis we find that $K$ has at most $n$ nonpiercing points (Theorem 5). In addition, the complications of $K-S$ are induced just by these nonpiercing points. Generally, at least two such points are required, for otherwise $n=0$ (Theorem 3).

A space $K$ as described above is called a crumpled cube. The boundary of $K$, denoted $\mathrm{Bd} K$, is defined by $\mathrm{Bd} K=S$, and the interior of $K$, denoted Int $K$, is defined by Int $K=K-\mathrm{Bd} K$. We also use the symbol $\mathrm{Bd}$ in another sense: if $M$ is a manifold with boundary, then $\mathrm{Bd} M$ denotes the boundary of $M$. This should not produce any confusion.

Let $K$ be a crumpled cube and $p$ a point in $\mathrm{Bd} K$. Then $p$ is a piercing point of $K$ if there exists an embedding $f$ of $K$ in the 3sphere $S^{3}$ such that $f(\mathrm{Bd} K)$ can be pierced with a tame arc at $f(p)$.

Let $U$ be an open subset of $S^{3}$. The limiting genus of $U$, denoted $L G(U)$, is the least nonnegative integer $n$ such that there exists a sequence $H_{1}, H_{2}, \ldots$ of compact 3-manifolds with boundary satisfying (1) $U=\cup H_{i}$, (2) $H_{i} \subset \operatorname{Int} H_{i+1}$, and (3) genus $\mathrm{Bd} H_{i}=n(i=1,2, \cdots)$. If no such integer exists, $L G(U)$ is said to be infinite. Throughout this paper the manifolds $H_{i}$ described above can be obtained with connected boundary, in which case $H_{i}$ is called a cube with $n$ holes.

Applications of the finite limiting genus condition are investigated in [6] and [14]. For any crumpled cube $K$ such that LG(Int $K)$ is finite and $\mathrm{Bd} K$ is locally peripherally collared from Int $K$, it is shown that $\mathrm{Bd} K$ is locally tame (from Int $K$ ) except at a finite set of points. Under the hypothesis of this paper, $\mathrm{Bd} K$ may be wild at every point; nevertheless, with a collapsing (in the sense of Whitehead [15]) argument comparable to [13, Th. 1], the problem of counting the nonpiercing points of $K$ is reduced to one in which the results of [6] and [14] apply.

A subset $X$ of the boundary of a crumpled cube $K$ is said to be semi-cellular in $K$ if for each open set $U$ containing $X$ there exists 
an open set $V$ such that $X \subset V \subset U$ and loops in $V-X$ are null homotopic in $U-X$. In the last section of this paper semi-cellular sets are discussed in order to characterize those sewings of two crumpled cubes which yield $S^{3}$, in case the limiting genus of one of the crumpled cubes is finite.

A simple closed curve $J$ is essential in an annulus $A$ if $J$ lies in $A$ and bounds no disk in $A$.

If $X$ is a set in a topological space, then $\mathrm{Cl} X$ denotes the closure of $X$.

\section{A cellularity criterion.}

Lemma 1. Let $H$ be a sphere with $n$ handles. Then there exists an integer $k(n)$ such that if $J_{1}, \cdots, J_{k(n)}$ are mutually exclusive simple closed curves in $H$, no one of which bounds a disk in $H$, then some pair $\left\{J_{r}, J_{s}\right\}$ bounds an annulus in $H$.

Proof. The number $k(n)=2$ is known to work if $n=1$. Otherwise, the proof proceeds by induction, using $k(n)=3 n-2$ whenever $n \geqq 2$.

THEOREM 2. Let $C$ be a crumpled cube such that $\mathrm{LG}(\operatorname{Int} C)=$ $n<\infty$. Then there exists a finite set $Q$ of points in $\mathrm{Bd} C$ such that for each open set $U \supset \mathrm{Bd} C$, each point of $\mathrm{Bd} C-Q$ has a neighborhood $V$ such that any loop in $V-\mathrm{Bd} C$ is null-homotopic in $U$ $\mathrm{Bd} C$.

Proof. Assume $n>0$. Using Lemma 1 we associate with a sphere with $n$ handles an integer $k(n)$. Let $k=\max \{3, k(n)\}$. Suppose $p_{1}, p_{2}, \cdots, p_{2 k}$ are points in $\mathrm{Bd} C$ and $U$ is an open set containing $\mathrm{Bd} C$. It suffices to show that one of these points has a neighborhood $V$ such that each loop in $V-\mathrm{Bd} C$ is nullhomotopic in $U-\mathrm{Bd} C$.

Step 1. Preliminary constructions. There exists a collection of mutually exclusive disks $D_{1}, \cdots, D_{2 k}$ on $\mathrm{Bd} C$ with $p_{i} \in \operatorname{Int} D_{i}(i=1$, $\cdots, 2 k)$. Furthermore, Bd $C$ contains another collection of mutually exclusive disks $E_{1}, \cdots, E_{k}$ such that for $i=1, \cdots, k$

$$
D_{2 i-1} \cup D_{2 i} \subset \operatorname{Int} E_{i} \text {. }
$$

We consider $C$ to be embedded in $S^{3}$ so that the closure of $S^{3}-C$ is a 3-cell $[8,10]$. We select a point $b$ of Int $C$ and construct arcs $B_{1}, \cdots, B_{2 k}$ such that (1) distinct $\operatorname{arcs} B_{i}$ and $B_{j}$ intersect only at the point $b,(2)$ the endpoints of $B_{i}$ are $b$ and $p_{i}$, and (3) $B_{i}$ is locally tame $\bmod p_{i}(\mathrm{i}=1, \cdots, 2 k)$. 
By Theorem 1 of [3] there exist pairwise disjoint annuli

$$
D_{1}^{*}, D_{2}^{*}, \cdots, D_{2 k}^{*}, E_{1}^{*}, E_{2}^{*}, \cdots, E_{k}^{*}
$$

in $S^{3}$ such that

(4) $\mathrm{Bd} D_{i}^{*} \supset \operatorname{Bd} D_{i}$ and $\mathrm{Bd} E_{j}^{*} \supset \operatorname{Bd} E_{j}$,

(5) $D_{i}^{*} \cap \mathrm{Bd} C \subset D_{i}$,

(5) $E_{j}^{*} \cap \mathrm{Bd} C \subset E_{j}-\left(D_{2 j-1} \cup D_{2 j}\right)$,

(6) $\left(\cup\left(\operatorname{Bd} D_{i}^{*}-\operatorname{Bd} D_{i}\right)\right) \cup\left(\cup\left(\operatorname{Bd} E_{j}^{*}-\operatorname{Bd} E_{j}\right)\right) \subset \operatorname{Int} C$,

(7) $D_{i}^{*}\left(E_{j}^{*}\right)$ is locally polyhedral $\bmod \mathrm{Bd} D_{i}\left(\mathrm{Bd} E_{j}\right)$, and

(8) $\left(\left(\cup D_{i}^{*}\right) \cup\left(\cup E_{j}^{*}\right)\right) \cap\left(\cup B_{i}\right)=\varnothing$.

If a surface approximating $\mathrm{Bd} C$ is to intersect the $D_{i}^{*}$ 's and $E_{j}^{*}$ 's properly, we must force it to lie very close to $\mathrm{Bd} C$. To do this, first we thicken certain subsets of $\mathrm{Bd} C$, thereby obtaining mutually exclusive open sets $W_{0}, W_{1}, \cdots, W_{3 k}$ such that

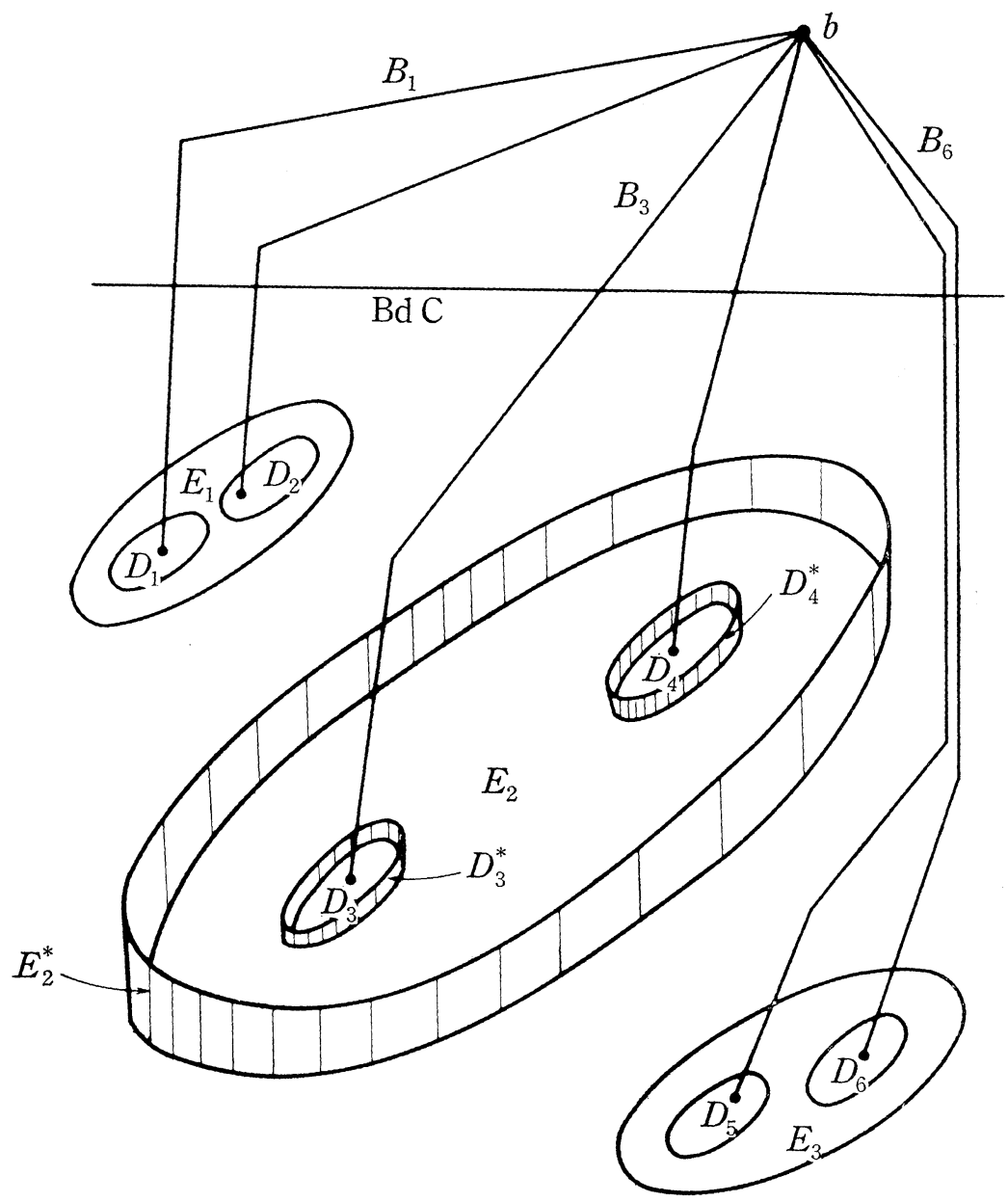

FIgURE 1 
(9) $W_{i} \cap C \subset U-\left(\left(\cup \mathrm{Bd} D_{i}^{*}\right) \cup\left(\cup \mathrm{Bd} E_{j}^{*}\right)\right)$,

(10) $W_{0} \supset \mathrm{Bd} C-\left(\left(\cup D_{i}\right) \cup\left(\cup E_{j}\right)\right)$,

(11) $W_{i} \supset \operatorname{Int} D_{i}(i=1, \cdots, 2 k)$,

(12) $W_{2 k+i} \supset$ Int $E_{i}-\left(D_{2 i-1} \cup D_{2 i}\right)(i=1, \cdots, k)$,

(13) $\left(\cup W_{j}\right) \cap B_{i}=W_{i} \cap B_{i}(i=1, \cdots, 2 k)$.

In addition, we require that $\mathrm{Bd} D_{i} \cap \mathrm{Cl} W_{s} \neq \varnothing$ only if $s=2 k+i$ or $s=i$ and $\mathrm{Bd} E_{j} \cap \mathrm{Cl} W_{s} \neq \varnothing$ only if $s=0$ or $s=2 k+j$. Then we construct a neighborhood $Y$ of $\mathrm{Bd} C-\cup W_{i}$ such that $Y \cap C \subset U$ and any arc in Int $C \cap\left(Y \cup\left(\cup W_{i}\right)\right)$ from a point of $W_{i}$ to a point of $W_{j}$ intersects all the annuli in between. For example, if $A$ is an arc from $W_{0}$ to $W_{1}$, then $A$ intersects both $E_{1}^{*}$ and $D_{1}^{*}$.

By hypothesis Int $C$ contains a cube with $n$ holes $M$ such that $C-\left(Y \cup\left(\cup W_{i}\right)\right) \subset \operatorname{Int} M$. Without loss of generality, we assume that $\mathrm{Bd} M$ is polyhedral and in general position with respect to

$\left(\cup \operatorname{Int} E_{j}^{*}\right) \cup\left(\cup \operatorname{Int} D_{i}^{*}\right)$.

Step 2. A special disk in $\mathrm{Bd} M$. Let $G$ denote the collection of those components of $\left.\mathrm{Bd} M \cap\left(\cup E_{j}^{*}\right) \cup\left(\cup D_{i}^{*}\right)\right)$ which are essential simple closed curves in any annulus $E_{j}^{*}$ or $D_{i}^{*}$. Each annulus $E_{j}^{*}\left(D_{i}^{*}\right)$ contains a curve in the collection $G$, because $\mathrm{Bd} M$ separates the components. of $\mathrm{Bd} E_{j}^{*}\left(\mathrm{Bd} D_{i}^{*}\right)$.

In the next paragraphs we show that at least one of the curves in $G$ bounds a disk in Bd $M$. Suppose the contrary. From Lemma 1 we find that $\mathrm{Bd} M$ contains an annulus $A$ such that $\mathrm{Bd} A=J_{r} \cup J_{s}$, where $J_{r}$ and $J_{s}$ are essential curves on $E_{r}^{*}$ and $E_{s}^{*}$, respectively, and $r \neq s$. This reduces to the case in which each component of Int $A \cap\left(\cup E_{j}^{*}\right)$ bounds a disk in $\cup E_{j}^{*}$. Assume $r \neq 1 \neq s$.

Case A. No component of $A \cap\left(\cup E_{j}^{*}\right)$ separates the components of $\mathrm{Bd} A$. Let $L$ be a simple closed curve in $S^{3}-\left(E_{1}^{*} \cup E_{r}^{*}\right)$ such that $L \cap C=B_{2} \cup B_{2 r}$. It follows from the constructions of Step 1 that each point of $L \cap A$ is separated (in $A$ ) from $J_{s}$ by a component of Int $A \cap\left(E_{1}^{*} \cup E_{r}^{*}\right)$; thus, by trading certain disks in Int $A$ for disks in $E_{1}^{*} \cup E_{r}^{*}$, we see that $J_{r}$ and $J_{s}$ are homotopic in $S^{3}-L$. But this is impossible, since $J_{r} \operatorname{links} L$ and $J_{s}$ does not.

Case B. Some component of $A \cap\left(\cup E_{j}^{*}\right)$ separates the components of $\mathrm{Bd} A$. By considering all components of $A \cap\left(\left(\cup E_{j}^{*}\right) \cup\left(\cup D_{i}^{*}\right)\right)$, we find that $A$ contains an annulus $A^{\prime}$ such that no curve in

$$
\text { Int } A^{\prime} \cap\left(\left(\cup E_{j}^{*}\right) \cup\left(D_{i}^{*}\right)\right)
$$

is essential in $A^{\prime}$ and $J_{r} \subset \mathrm{Bd} A^{\prime}$. Let $J^{\prime}$ denote the other component of $\mathrm{Bd} A^{\prime}$, and without loss of generality assume that $J^{\prime} \cap D_{2 r}^{*}=\varnothing$. 
Let $L^{\prime}$ be a simple closed curve in $S^{3}-\left(\left(\cup E_{j}^{*}\right) \cup\left(\cup D_{i}^{*}\right)\right)$ such that $L^{\prime} \cap C=B_{2} \cup B_{2 r}$. Each point of $L^{\prime} \cap A^{\prime}$ is separated in $A^{\prime}$ from either $J_{r}$ or $J^{\prime}$ by $\operatorname{Int} A^{\prime}\left(\left(\cup E_{j}^{*}\right) \cup\left(\cup D_{i}^{*}\right)\right)$, and each curve of this intersection bounds disks in both $A^{\prime}$ and $\left(\cup E_{j}^{*}\right) \cup\left(\cup D_{i}^{*}\right)$. Hence, by the usual disk trading, we see that $J_{r}$ is homotopic to $J^{\prime}$ in $S^{3}-L^{\prime}$. Again this leads to a contradiction, for $J_{r}$ links $L^{\prime}$; on the other hand, $J^{\prime}$ either is contained in $D_{2 r-1}^{*}$ or is an inessential curve in some $E_{j}^{*}$, which implies that $J^{\prime}$ does not link $L^{\prime}$.

Neither of the two cases can occur. Consequently, some simple closed curve $J$ in the collection $G$ bounds a disk in Bd $M$.

Step 3. A neighborhood $V$ of one of the points $p_{i}$. Corresponding to one of the points, say $p_{1}$, there exists a disk $D \subset \mathrm{Bd} M$ such that $\mathrm{Bd} D$ is an essential curve in $D_{1}^{*}$, but each component of Int $D \cap\left(\cup D_{i}^{*}\right)$ bounds a disk in $\cup D_{i}^{*}$. Repeating this process, it follows that for one of the $p_{i}$ 's, say $p_{1}$ again, and for each open set $U^{\prime}$ containing $\mathrm{Bd} C$, there exists a polyhedral disk $E$ in $U^{\prime} \cap \operatorname{Int} C$ such that $\mathrm{Bd} E$ is an essential simple closed curve on $D_{1}^{*}$ but each component of (Int $\left.E \cap\left(\cup D_{i}^{*}\right)\right)$ bounds a disk in $\cup D_{i}^{*}$.

To find the desired open set in $C$, let $V^{\prime}$ be a spherical neighborhood of $p_{1}$ such that $V^{\prime} \cap C \subset W_{1}$, and define $V=V^{\prime} \cap C$. For any loop $L$ in $V-\operatorname{Bd} C$, another linking argument shows that $L$ is separated from $\mathrm{Bd} C$ (in $V$ ) by some disk $E \subset U$ as described above. Since $L$ is contractible in $V^{\prime}$, it follows from [5, Lemma 1] that $L$ is contractible in $U-\mathrm{Bd} C$. This completes the proof.

TheOREM 3. Suppose $C$ is a crumpled cube such that $\mathrm{LG}(\operatorname{Int} C)<$ $\infty$ and $C$ contains at most one nonpiercing point. Then $\operatorname{Int} C$ is an open 3-cell.

Proof. Assume $C$ is embedded in $S^{3}$ so that the closure of $S^{3}-C$ is a 3-cell $K[8,10]$. Equivalently, we show that $K$ is a cellular subset of $S^{3}$.

Let $Q$ denote the finite set of points of $\mathrm{Bd} C$ given by Theorem $2, p$ the nonpiercing point of $C$ (the argument when $C$ has no nonpiercing point is essentially the same), and $U$ an open set containing $K$. There exists an open set $V$ containing $K$ such that loops in $V-K$ are null-homotopic in $U-($ Int $K \cup p)$. Let $f$ be a map of a disk $\Delta$ into $U-(\operatorname{Int} K \cup p)$ such that $f(\operatorname{Bd} \Delta) \subset V-K$. It follows from [12, Th. 2] and techniques of [2, Th. 4.2] that $f$ can be adjusted slightly at points of Int $\Delta$ so that $f(\Delta) \cap \mathrm{Bd} C$ is 0 -dimensional and $f(\Delta) \cap Q=\varnothing$. Finally, there exists a finite number of mutually exclusive simple closed curves $S_{1}, \cdots S_{k}$ in $\Delta$ whose union separates $\mathrm{Bd} \Delta$ from $f^{-1}(f(\Delta)) \cap \mathrm{Bd} C$ ) and such that $f \mid S_{i}$ is null homotopic in 
$U-K(i=1, \cdots, k)$. This implies that $f \mid \operatorname{Bd} \Delta$ extends to a map of $\Delta$ into $U-K$. According to McMillan's Cellularity Criterion [11, Th. $\left.1^{\prime}\right], K$ is a cellular subset of $S^{3}$.

3. Topological collapsing. The following result generalizes Theorem 1 of [13]. The argument below necessarily differs from McMillan's, since we have no mapping criterion to determine the finite limiting genus condition.

Theorem 4. Suppose $K$ is a finite connected simplicial complex, $L$ a subcomplex of $K$ such that $K$ collapses to $L$, and $h$ a homeomorphism of $K$ into $S^{3}$ such that $\operatorname{LG}\left(S^{3}-h(K)\right)=n$. Then

$$
\mathrm{LG}\left(S^{3}-h(L)\right) \leqq n
$$

Proof. It is sufficient to show that the result holds if $L$ is obtained from $K$ by a single elementary collapse. Suppose that $\sigma$ is a principal simplex of $K, \tau$ is a proper face of $\sigma$ such that $\tau$ is a proper face of no other simplex in $K$, and

$$
L=K-\operatorname{Int} \sigma-\operatorname{Int} \tau \text {. }
$$

We consider the case when $\sigma$ is a 3 -simplex, because the applications of Theorem 4 in this paper can be viewed as involving collapses of this type only; for the remaining cases a similar argument applies.

Let $U$ be an open subset of $S^{3}$ containing $h(L)$. There exists a neighborhood $U^{*}$ of $h(L)$ in $U$ such that some component $Z$ of $h(\sigma)-U^{*}$ contains $h(\sigma)-U$. Using [4, Th. 4] we find a tame disk $D$ in $U^{*}-h(L)$ such that $\mathrm{Bd} D \cap h(K)=\varnothing$ and exactly one of the components of $D \cap h(\sigma)$ separates $Z$ from $h(L \cap \sigma)$ in $h(\sigma)$.

There exists a neighborhood $W$ of $h(K)$ such that $W \cap \operatorname{Bd} D=\varnothing$ and $W$ can be deformed to $h(K)$ in $S^{3}-\mathrm{Bd} D$ by a homotopy keeping $h(K)$ pointwise fixed. For each point $x$ in $U \cap h(K)$ define an open set $N_{x}$ as

$$
N_{x}=\left\{y \in S^{3} \mid \rho(x, y)<\rho(x, \operatorname{Bd} U \cup \operatorname{Bd} W)\right\}
$$

and for each point $x$ in $h(\sigma)-U$ define $N_{x}$ as

$$
N_{x}=\left\{y \in S^{3} \mid \rho(x, y)<\rho(x, D \cup \operatorname{Bd} W)\right\} \text {. }
$$

Then let $V=\bigcup_{x \in h(K)} N_{x}$.

Claim. $D \cap V$ separates $Z$ from $h(L)$ in $V$, and $U$ contains the component $Y$ of $V-D$ that contains $h(L)$.

Suppose there exists an arc $\alpha$ in $V-D$ from a point of $Z$ to a 
point of $h(L)$. Then $\alpha$ is homotopic in $S^{3}-\mathrm{Bd} D$ (with endpoints fixed) to a path $\alpha^{\prime}$ in $h(K)$, and $\alpha^{\prime}$ is homotopic in $h(K)$ (with endpoints fixed) to a path $\alpha^{*}$ such that $\alpha^{*} \cap D$ consists of a finite set of points at which $\alpha^{*}$ pierces $D$. But then the number of such points must be even, contradicting the separation properties of $D$ in $h(K)$.

To establish the other part of the claim, suppose there exists a point $y$ in $Y-U$. Then $y \in N_{x}$ for some $x$ in $h(\sigma)-U$. Let $A$ be the straight line segment from $y$ to $x$ in $N_{x}$, and let $B$ denote an arc from $y$ to $h(L)$ in $Y$. Since $A \cup B$ does not intersect $D$, deforming $A \cup B$ to a path in $h(K)$ leads to a contradiction as before. This completes the proof of the claim.

By hypothesis $S^{3}-h(K)$ contains a polyhedral cube with $n$ holes $H$ such that Int $H \supset S^{3}-V$. We adjust $H$ slightly so that $\operatorname{Bd} H \cap D$ consists of a finite number of simple closed curves. Note that $D \cup$ (Bd $H \cap U$ ) separates $h(L)$ from $h(\sigma)-U$ (in $S^{3}$ ). Thus, the unicoherence of $S^{3}-D$ implies that some component $F$ of $\mathrm{Bd} H-D$, where $F \subset U$, separates $h(L)$ from $h(\sigma)-U$ in $S^{3}-D$.

We observe that $\mathrm{Cl} F$ is a disk with $k(k \leqq n)$ handles and (possibly) some holes. By attaching disks to $\operatorname{Bd} F$ near $D$, we see that $F$ is contained in a sphere with $k$ handles $S_{k}$ in $\mathrm{C} 1\left(S^{3}-h(L)\right)$ and that $S_{k}$ bounds a cube with $k$ holes $M$ satisfying

$$
S^{3}-U \subset M \subset S^{3}-h(L) \text {. }
$$

This implies that $\mathrm{LG}\left(S^{s}-h(L)\right) \leqq n$.

\section{The number of nonpiercing points.}

THEOREM 5. If $C$ is a crumpled cube such that $\mathrm{LG}(\operatorname{Int} C)=n$ $(1 \leqq n<\infty)$, then $C$ has at most $n$ nonpiercing points.

Proof. Suppose to the contrary that $C$ contains at least $n+1$ nonpiercing points $p_{1}, \cdots, p_{n+1}$. As before we assume $C$ is embedded in $S^{3}$ so that the closure of $S^{3}$ of $S^{3}-C$ is a 3-cell $H[8,10]$. Let $h$ denote a homeomorphism of a 3 -simplex $\Delta^{3}$ onto $H$.

Some triangulation $K$ of $\Delta^{3}$ collapses to a subcomplex $L$ such that $h(L)$ is a 3-cell locally tame except at $p_{1}, \cdots, p_{k+1}$; thus, each point $p_{i}$ is a nonpiercing point of $\mathrm{C} 1\left(S^{3}-h(L)\right)$. Theorem 4 gives that $\mathrm{LG}\left(S^{3}-h(L)\right) \leqq n$. This leads to a contradiction, however, for either [6, Th. 2] or [14, Th. 1] implies that $\mathrm{C} 1\left(S^{3}-h(L)\right)$ has at most $n$ nonpiercing points.

CoRollary. If $C$ is a crumpled cube such that $\mathrm{LG}(\operatorname{Int} C) \leqq 1$, then Int $C$ is an open 3-cell. 
The techniques used to prove Theorem 5 can be reapplied to obtain the following result.

THEOREM 6. If $H$ is a cube with $k$ handles in $S^{3}$ and

$$
\mathrm{LG}\left(S^{3}-H\right)=n(1 \leqq n<\infty),
$$

then $\mathrm{Bd} H$ is pierced by a tame arc at all but (at most) $n-k$ of its points.

To describe the number of nonpiercing points precisely requires some additional definitions. Let $A$ be an arc in $S^{3}$ locally tame modulo an endpoint $p$. The local enveloping genus of $A$ at $p$, denoted LEG $(A, p)$, is the smallest nonnegative integer $r$ (if there is no such integer $r, \operatorname{LEG}(A, p)=\infty)$ such that there exist arbitrarily small neighborhoods of $p$, each of which is bounded by a surface of genus $r$ (a sphere with $r$ handles) that intersects $A$ at exactly one point. Chapter 4 of [14] gives illustrations of $\operatorname{arcs} A_{n}$, each locally tame $\bmod$ an endpoint $p_{n}$, such that $\operatorname{LEG}\left(A_{n}, p_{n}\right)=n(n=1,2, \cdots, \infty)$.

Let $B=\left\{(x, y, z) \in E^{3} \mid x^{2}+y^{2}+z^{2} \leqq 1\right\}$. Let $f$ be a homeomorphism of $B$ onto a 3-cell $C$ in $S^{3}$, and $p$ a point of $\mathrm{Bd} C$. The local enveloping genus of $C$ at $p$, denoted $\operatorname{LEG}(C, p)$, is defined by

$$
\operatorname{LEG}(C, p)=\operatorname{LEG}(f(\alpha), p),
$$

where $\alpha$ is the line segment in $B$ from the origin to $f^{-1}(p)$.

THeOREM 7. If $C$ is a 3-cell in $S^{3}$ such that $\mathrm{LG}\left(S^{3}-C\right)=n$ $(2 \leqq n<\infty)$ and $p_{1}, \cdots, p_{k}$ are the nonpiercing points of $S^{3}-\operatorname{Int} C$, then

$$
n=\sum_{i=1}^{k} \operatorname{LEG}\left(C, p_{i}\right) \text {. }
$$

Proof. As in the proof of Theorem 5, let $h$ be a homeomorphism of a 3-simplex $\Delta^{3}$ onto $C$. Some triangulation of $\Delta^{3}$ collapses to a subcomplex $L$ such that $h(L)$ is a 3-cell locally tame modulo $\cup p_{i}$. It follows from the definition of local enveloping genus that the subcomplex $L$ can be chosen to satisfy

$$
\operatorname{LEG}\left(C, p_{i}\right)=\operatorname{LEG}\left(h(L), p_{i}\right) \quad(i=1, \cdots, k) .
$$

Since $\mathrm{LG}\left(S^{3}-h(L)\right) \leqq n$, Theorem 6 of [14] implies

$$
n \geqq \Sigma \operatorname{LEG}\left(h(L), p_{i}\right)=\Sigma \operatorname{LEG}\left(C, p_{i}\right) \text {. }
$$

Let $U$ be an open set containing $C$. To establish the inequality in the other direction, we shall find pairwise disjoint disks with handles 
$G_{1}, \cdots, G_{k}$ in $U-\cup p_{i}$ subject to the following conditions: the number of handles on $G_{i}$ is bounded by $\operatorname{LEG}\left(C, p_{i}\right), \mathrm{Bd} G_{i}$ bounds an annulus $A_{i}$ in $G_{i}$ such that $G_{i}^{\prime}=\mathrm{Cl}\left(G_{i}-A_{i}\right)$ is contained in $U-C$, Int $A_{i} \cap \mathrm{Bd} C$ is contained both in a null sequence of pairwise disjoint disks in $\mathrm{Bd} C-\cup p_{i}$ and in a null sequence of such disks in $\operatorname{Int} A_{i}$, and $\cup \mathrm{Bd} G_{i}$ bounds a disk with $(k-1)$ holes in $\mathrm{Bd} C-\cup p_{i}$. Furthermore, $G_{i}$ can be obtained arbitrarily close to $p_{i}$. Thus, in the next two paragraphs we describe how to find one such surface $G_{1}$ near $p_{1}$.

In $\mathrm{Bd} C$ there exists a Sierpinski curve $X$ locally tame mod $p_{1}$ and containing $p_{1}$ in its inaccessible part. By removing a null sequence of nice 3-cells from $C$ we obtain a 3-cell $C^{*}$ such that $C^{*} \cap \mathrm{Bd} C=X$ and $C^{*}$ is locally tame $\bmod p_{1}$. It follows from the definition of local enveloping genus that arbitrarily close to $p_{1}$ is a surface $H$ such that $H \cap C^{*}$ is a disk $D$, with $D \cap \mathrm{Bd} C^{*}=\mathrm{Bd} D$, and $p_{1}$ lies interior to the small disk on $\mathrm{Bd} C^{*}$ bounded by $\mathrm{Bd} D$. Adjust $H$ near $\mathrm{Bd} C^{*}$ so that $\mathrm{Bd} D$ lies in the inaccessible part of $X$. Without moving any point of $D$ adjust $H$ further so that the nondegenerate components of $(H-D) \cap \mathrm{Bd} C$ comprise a null sequence of simple closed curves and that $(H-D) \cap C^{*}=\varnothing[4$, Th. 4]. Hence,

$$
(H-D) \cap X=\varnothing \text {. }
$$

Now consider the component $K$ of $H-C$ whose closure contains $\mathrm{Bd} D$. Associate with each simple closed curve $S_{j}$ of $(\mathrm{Bd} K-\mathrm{Bd} D)$ a disk $F_{j}$ in $C-C^{*}$ such that

(1) $F_{j} \cap \mathrm{Bd} C=\mathrm{Bd} F_{j}=S_{j}$,

(2) $F_{j} \cap F_{k}=\varnothing$ if $S_{j} \cap S_{k}=\varnothing$,

(3) $\lim _{j \rightarrow \infty} \operatorname{diam} F_{j}=0$.

Define $G_{1}=\left(\cup F_{j}\right) \cup \mathrm{C} 1 K$. Then $G_{1}$ is a disk with handles, and the number of handles is bounded by $\operatorname{LEG}\left(C, p_{1}\right)$. Note that $\operatorname{Bd} G_{1}=\operatorname{Bd} D$. Since components of $\left(G_{1}-\mathrm{Bd} G_{1}\right) \cup C$ are either arcs or points, we can readily obtain an annulus $A_{1}$ in $G_{1}$ such that $\mathrm{Bd} A_{1}$ contains $\mathrm{Bd} G_{1}$ and Int $A_{1}$ contains $\left(G_{1}-\mathrm{Bd} G_{1}\right) \cap C$, and now the remaining requirements on $G_{1}$ must be satisfied.

Applying Theorem 2 and techniques from the proof of Theorem 3 , we find a map $f$ of a disk with $(k-1)$ holes $E$ into $U-C$ such that

$$
f(E) \cap G_{i}^{\prime}=f(\operatorname{Bd} E) \cap G_{i}^{\prime}=\operatorname{Bd} G_{i}^{\prime} \quad(i=1, \cdots, k)
$$

and $f$ has no singularities near Bd $E$. According to [9, Lemma 1] there exists a homeomorphism $f^{\prime}$ of $E$ into $U-C$ such that

$$
f^{\prime}(E) \cap G_{i}^{\prime}=f^{\prime}(\operatorname{Bd} E) \cap G_{i}^{\prime}=\operatorname{Bd} G_{i}^{\prime} \quad(i=1, \cdots, k) .
$$

Thus, if $S$ denotes $f^{\prime}(E) \cup\left(\cup G_{i}^{\prime}\right), S$ is a sphere with handles, and 
the number of handles is bounded by $\Sigma \operatorname{LEG}\left(C, p_{i}\right)$. Moreover, $S$ can be obtained so as to separate $S^{3}-U$ from $C$. Finally, since $U$ is an arbitrary open set, we have that

$$
n \leqq \sum \operatorname{LEG}\left(C, p_{i}\right)
$$

5. Semi-cellular subsets.

THEOREM 8. Suppose $C$ is a crumpled cube such that

$$
2 \leqq \mathrm{LG}(\operatorname{Int} C)<\infty,
$$

and $X$ is a nonseparating subcontinuum of $\mathrm{Bd} C$ containing only piercing points of $C$. Then $X$ is semi-cellular in $C$.

Proof. Let $p_{1}, \cdots, p_{k}$ denote the nonpiercing points of $C$, and $D$ a disk in $\mathrm{Bd} C-\cup p_{i}$ whose interior contains $X$. If $C$ is embedded in $S^{3}$ so that $\mathrm{Cl}\left(S^{3}-C\right)$ is a 3-cell $K$, then $K$ collapses to a 3-cell $K^{\prime}$ which is locally tame mod $\left(D \cup p_{1}\right)$, with $p_{1}$ a nonpiercing point of $S^{3}-$ Int $K^{\prime}=C^{\prime}$. According to Theorem 4, LG(Int $\left.C^{\prime}\right)<\infty$. Since each point of $D$ is a piercing point of $C^{\prime}$, it follows from Theorem 3 that Int $C^{\prime}$ is an open 3-cell. Then $X$ is semi-cellular in $C^{\prime}$ [7, Lemma 2.7]; clearly $X$ must also be semi-cellular in $C$.

Theorem 8 can be applied to characterize those sewings of two crumpled cubes which yield $S^{3}$, when one of the crumpled cubes has finite limiting genus. With minor changes, such as in the references to the number of nonpiercing points, we can use the proof of $[7, \mathrm{Th}$. 5.7] to prove Theorem 9 .

THeonem 9. Suppose $C_{1}$ and $C_{2}$ are crumpled cubes, $h$ is a homeomorphism of $\mathrm{Bd} C_{1}$ to $\mathrm{Bd} C_{2}$, and $\mathrm{LG}\left(\operatorname{Int} C_{2}\right)<\infty$. Then $C_{1} \mathrm{U}_{h} C_{2}=$ $S^{3}$ if and only if each nonpiercing point of $C_{1}$ is identified by $h$ with a piercing point of $C_{2}$.

\section{REFERENCES}

1. R. H. Bing, Approximating surfaces from the side, Ann. of Math. (2) 65 (1957), 456-483.

2. - Pushing a 2-sphere into its complement, Michigan Math. J. 11 (1964), $33-45$.

3. Improving the side approximation theorem, Trans. Amer. Math. Soc. 116 (1965), 511-525.

4. - Improving the intersection of lines and surfaces, Michigan Math. J. 14 (1967), 155-159.

5. C. E. Burgess, Characterizations of tame surfaces in $E^{3}$, Trans. Amer. Math. Soc. 114 (1965), 80-97.

6. R. J. Daverman, Non-homeomorphic approximations of manifolds with surfaces of 
bounded genus (to appear in Duke Math. J.)

7. R. J. Daverman and W. T. Eaton, Universal crumpled cubes (to appear).

8. N, Hosay, The sum of a real cube and a crumpled cube is $S^{3}$, Notices Amer. Math. Soc. 10 (1963), 666. See also errata 11 (1964), 152.

9. H. W. Lambert, Mapping cubes with holes onto cubes with handles, Illinois J. Math. 13 (1969), 606-615.

10. L. L. Lininger, Some results on crumpled cubes, Trans. Amer. Math. Soc. 118 (1965), 534-549.

11. D. R. McMillan, Jr., A criterion for cellularity in a manifold, Ann. of Math. (2) 79 (1964), 327-337.

12. Some topological properties of piercing points, Pacific J. Math. 22 (1967), $313-322$.

13. - Piercing a disk along a cellular set, Proc. Amer. Math. Soc. 19 (1968), 153-157.

14. M. D. Taylor, An upper bound for the number of wild points on a 2-sphere, $\mathrm{Ph}$. D. Thesis, Florida State University, 1969.

15. J. H. C. Whitehead, Simplicial spaces, nuclei and m-groups, Proc. London Math. Soc. 45 (1939), 243-327.

Received June 4, 1969, and in revised form November 14, 1969. This paper supported in part by NSF Grant GP-8888.

University of Tennessee 



\section{PACIFIC JOURNAL OF MATHEMATICS}

EDITORS

H. SAMELSON

Stanford University

Stanford, California 94305

RichaRd PIERCE

University of Washington

Seattle, Washington 98105
J. DUGUNDJI

Department of Mathematics

University of Southern California

Los Angeles, California 90007

RICHARD ARENS

University of California

Los Angeles, California 90024

\section{ASSOCIATE EDITORS}

E. F. BECKENBACH

B. H. NeumanN

F. WOLF

K. YOSHIDA

\section{SUPPORTING INSTITUTIONS}

UNIVERSITY OF BRITISH COLUMBIA

CALIFORNIA INSTITUTE OF TECHNOLOGY

UNIVERSITY OF CALIFORNIA

MONTANA STATE UNIVERSITY

UNIVERSITY OF NEVADA

NEW MEXICO STATE UNIVERSITY

OREGON STATE UNIVERSITY

UNIVERSITY OF OREGON

OSAKA UNIVERSITY

UNIVERSITY OF SOUTHERN CALIFORNIA
STANFORD UNIVERSITY

UNIVERSITY OF TOKYO

UNIVERSITY OF UTAH

WASHINGTON STATE UNIVERSITY

UNIVERSITY OF WASHINGTON

AMERICAN MATHEMATICAL SOCIETY CHEVRON RESEARCH CORPORATION TRW SYSTEMS

NAVAL WEAPONS CENTER 


\section{Pacific Journal of Mathematics}

May, 1970

Johan Aarnes, Edward George Effros and Ole A. Nielsen, Locally compact

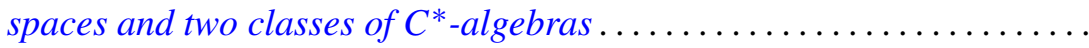

Allan C. Cochran, R. Keown and C. R. Williams, On a class of topological

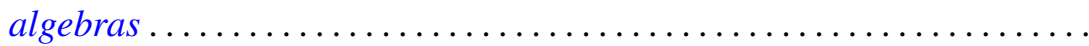

John Dauns, Integral domains that are not embeddable in division rings ....

Robert Jay Daverman, On the number of nonpiercing points in certain

crumpled cubes.....................................

Bryce L. Elkins, Characterization of separable ideals ................

Zbigniew Fiedorowicz, A comparison of two naturally arising uniformities

on a class of pseudo-PM spaces ...........................

Henry Charles Finlayson, Approximation of Wiener integrals of functionals

continuous in the uniform topology ........................

Theodore William Gamelin, Localization of the corona problem ...........

Alfred Gray and Paul Stephen Green, Sphere transitive structures and the

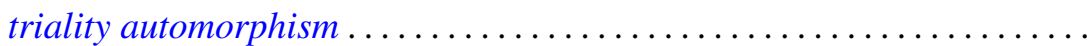

Charles Lemuel Hagopian, On generalized forms of aposyndesis ..........

J. Jakubík, On subgroups of a pseudo lattice ordered group ...............

Cornelius W. Onneweer, On uniform convergence for Walsh-Fourier

series..................................

Stanley Joel Osher, On certain Toeplitz operators in two variables ...

Washek (Vaclav) Frantisek Pfeffer and John Benson Wilbur, On the

measurability of Perron integrable functions............

Frank J. Polansky, On the conformal mapping of variable regions...

Kouei Sekigawa and Shûkichi Tanno, Sufficient conditions for a Riemannian manifold to be locally symmetric ...................

James Wilson Stepp, Locally compact Clifford semigroups ....

Ernest Lester Stitzinger, Frattini subalgebras of a class of solvable Lie

algebras ................................

George Szeto, The group character and split group algebras...

Mark Lawrence Teply, Homological dimension and splitting torsion

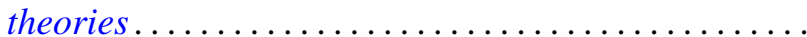

David Bertram Wales, Finite linear groups of degree seven. II ...

Robert Breckenridge Warfield, Jr., An isomorphic refinement theorem for

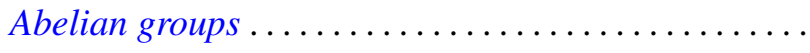

James Edward West, The ambient homeomorphy of an incomplete subspace

of infinite-dimensional Hilbert spaces................

Peter Wilker, Adjoint product and hom functors in general topology ...

Daniel Eliot Wulbert, A note on the characterization of conditional 